\title{
New algorithms for solving zero-sum stochastic games
}

\author{
Miquel Oliu-Barton*
}

December 17, 2019

\begin{abstract}
Zero-sum stochastic games, henceforth stochastic games, are a classical model in game theory in which two opponents interact and the environment changes in response to the players' behavior. The central solution concepts for these games are the discounted values and the value, which represent what playing the game is worth to the players for different levels of impatience. In the present manuscript, we provide algorithms for computing exact expressions for the discounted values and for the value, which are polynomial in the number of pure stationary strategies of the players. This result considerably improves all the existing algorithms, including the most efficient one, due to Hansen, Koucký, Lauritzen, Miltersen and Tsigaridas (STOC 2011).
\end{abstract}

\section{Introduction}

\subsection{Motivation}

Zero-sum stochastic games, henceforth stochastic games, were introduced by Shapley [20] in 1953 in order to model the dynamic interaction between two opponents. The theory of stochastic games and its applications have been studied in several scientific disciplines, including economics, operations research, evolutionary biology, and computer science. In addition, mathematical tools that were used and developed in the study of stochastic games are used by mathematicians and computer scientists in other fields. Computer scientists refer to stochastic games as concurrent games, a terminology that was introduced to distinguish these games from the so-called turned-based (or simple) stochastic games, where the players alternate moves.

Stochastic games generalize matrix games and Markov decision problems; they are played over a finite set of states, and to each state corresponds a matrix game. Stochastic games are played in stages. At each stage $m \geq 1$, a stage reward $g_{m}$ is produced, which depends on the current state $k_{m}$, commonly observed by the players, and on the

\footnotetext{
*Université Paris-Dauphine, PSL Research University, CNRS, CEREMADE, Paris, France.
} 
current pair of actions $\left(i_{m}, j_{m}\right)$ chosen by the players. The game is zero-sum, in the sense that Player 1 receives $g_{m}$, while Player 2 receives $-g_{m}$. A $\lambda$-discounted stochastic game is one where Player 1 maximizes the expectation of the normalized $\lambda$-discounted sum $\sum_{m \geq 1} \lambda(1-\lambda)^{m-1} g_{m}$ for some discount rate $\lambda \in(0,1]$, while Player 2 minimizes the same amount. The case where the discount rate is close to 0 is of particular importance, as it stands for the case where the players are patient. Alternatively, the interaction between patient players can be modeled by an undiscounted stochastic game, that is: one in which Player 1 maximizes the expectation of $\lim _{i n f} \inf _{T \rightarrow \infty} \frac{1}{T} \sum_{m=1}^{T} g_{m}$, while Player 2 minimizes the same amount.

The central solution concept for zero-sum games is its value. When it exists, the value is the maximal amount that each player can obtain in expectation regardless of her opponent's behavior. The value of the $\lambda$-discounted stochastic game is often referred to as its $\lambda$-discounted value, while the value of the undiscounted stochastic game is referred to as its value. In the present manuscript, we propose new algorithms for computing the discounted values and the value of any stochastic game, which are polynomial in the number of pure stationary strategies of the game, that is: strategies that depend only on the current state. More precisely, for a stochastic game with $n$ states and $p$ actions available at each state, we provide explicit bounds which are polynomial in $p^{n}$. These results considerably improve all prior algorithms for computing the discounted values and the value of a stochastic game. In particular, they improve the best of them, due to Hansen, Koucký, Lauritzen, Miltersen and Tsigaridas [10], where the bounds are polynomial in $p$ and $2^{n^{n^{2}}}$.

Notation. In the sequel, we denote by $K=\{1, \ldots, n\}$ the set of states, for some $n \in \mathbb{N}$. For any initial state $1 \leq k \leq n$ any discount rate $\lambda \in(0,1]$, the $\lambda$-discounted value and the value of the stochastic game starting at $k$ are denoted, respectively, by $v_{\lambda}^{k}$ and $v^{k}$. We also set $v_{\lambda}:=\left(v_{\lambda}^{1}, \ldots, v_{\lambda}^{n}\right) \in \mathbb{R}^{n}$ and $v:=\left(v^{1}, \ldots, v^{n}\right) \in \mathbb{R}^{n}$.

\subsection{State of the art}

In his seminal paper, Shapley [20] defined stochastic and proved that these games have a $\lambda$-discounted value for each $\lambda \in(0,1]$, and that both players have optimal stationary strategies, that is, strategies that depend only on the current state. Furthermore, a characterization was obtained for the vector of values $v_{\lambda}$, as the unique fixed point of an operator from $\mathbb{R}^{n}$ to $\mathbb{R}^{n}$ which is contracting for the $L^{\infty}$ norm. Blackwell and Ferguson [5] considered a particular stochastic game, the so-called "Big Match", and proved that the existence of the value and the equality to $\lim _{\lambda \rightarrow 0} v_{\lambda}=v$. Their result was then extended by Kohlberg [14] to the class of absorbing games, that is, a class of stochastic games in which there is at most one transition between states. For general stochastic games, the convergence of the discounted values, as $\lambda$ goes to 0 , was proved by Bewley and Kohlberg [3], building on Shapley's characterization of the discounted values and on Tarski-Seidenberg elimination theorem from semi-algebraic geometry. The existence of the value and the equality $v=\lim _{\lambda \rightarrow 0} v_{\lambda}$, were proved by Mertens and Neyman [16]. An explicit characterization of the value was recently obtained by Attia and Oliu-Barton [1]. 
Whether the value of a finite stochastic game can be computed in polynomial time is a famous open problem in computer science. This problem is intriguing because the simpler class of simple stochastic games is both NP and co-NP, and several famous problems with this property have eventually been shown to be polynomial-time solvable, such as primality testing or linear programming. (A simple stochastic game is one where, for each state, the transition function depends on one player's action only.) The known algorithms fall into two categories: decision procedures for the first order theory of the reals, such as [7, 8, 22], and value or strategy iteration methods, such as $[11,18]$. All of them are worst-case exponential in the number of states or in the number of actions. Hansen, Ibsen-Jensen and Miltersen [9] proved that no value or strategy iteration algorithm can ever achieve a polynomial bound. Recently, Hansen, Koucký, Lauritzen, Miltersen and Tsigaridas [10] obtained a remarkable improvement using the machinery of real-algebraic geometry in a more indirect manner: they provided an algorithm which, for any fixed number of states, is polynomial in the number of actions. However, the dependence on the number of states is an implicit double exponential expression, which is problematic in terms of practical computations. In their own words (page 3 of [10]): "the exponent in the polynomial time bound is $O(n)^{n^{2}}$, i.e., the complexity is doubly exponential in n", from which they claim that "getting a better dependence on $n$ is a very interesting open problem".

\subsection{Main results}

In the present paper, we propose a new method for computing the $\lambda$-discounted value and the value of a stochastic game. Unlike all prior works, we build on the new characterizations that were obtained by Attia and Oliu-Barton [1]. Our algorithms are polynomial in the number of actions, for any fixed number of states, but the dependence on the number of states is explicit and simply exponential. Equivalently, our algorithms are polynomial in the number of pure stationary strategies, that is, strategies that depend only on the current state. This improvement opens up the path for actually solving stochastic games in practice. An important ingredient in our work is the following continuity result: for any $\varepsilon>0$, we provide an explicit discount rate $\lambda_{\varepsilon} \in(0,1]$ whose bit-size is is polynomial in the number of pure stationary strategies and in $\log \varepsilon$, and so that $\left|v_{\lambda}^{k}-v^{k}\right| \leq \varepsilon$ for all $\lambda \in\left(0, \lambda_{\varepsilon}\right)$ and $1 \leq k \leq n$.

\subsection{Organisation of the paper}

In Section 2, we introduce the model (Section 2.1), state our main results formally (Section 2.2), and recall some relevant results from the literature (Section 2.3). Section 3 is devoted to proof the results that do not involve algorithms, namely, some algebraic properties for $v_{\lambda}^{k}$ and $v^{k}$, and a bound for the discount rate which ensures that the two are close to each other. Section 4 is devoted to the algorithms. More precisely, we start by recalling some classical algorithms (Section 4.1). Next, we present two algorithms: one which outputs a crude approximation of $v_{\lambda}^{k}$, and one which outputs an exact expression for $v_{\lambda}^{k}$ (Section 4.2). Similarly, Section 4.3 is devoted to the computation of a crude approximation, and of an exact expression, for $v^{k}$. Finally, we provide an example in Section 4.4 in order to illustrate our results. 


\section{Stochastic games}

We now introduce the model of stochastic games, and some basic facts. For a more detailed presentation of stochastic games, see for instance Sorin [23, Chapter 5] and Renault [19].

\subsection{Model and notation}

We start by introducing some notation that will be used throughout the paper.

- For each finite set $E$, we denote its cardinality by $|E|$ and the set of probability distributions over $E$ by $\Delta(E)=\left\{f: E \rightarrow[0,1], \sum_{e \in E} f(e)=1\right\}$.

- We denote by $n$ the number of states.

- $I^{1}, \ldots, I^{n}$ and $J^{1}, \ldots, J^{n}$ denote $2 n$ fixed finite sets of actions.

- We set $I:=I^{1} \times \cdots \times I^{n}$ and $J:=J^{1} \times \cdots \times J^{n}$.

- We set $d:=\min (|I|,|J|)$.

- We set $X:=\Delta\left(I^{1}\right) \times \cdots \times \Delta\left(I^{n}\right)$ and $Y:=\Delta\left(J^{1}\right) \times \cdots \times \Delta\left(J^{n}\right)$.

- For any $\alpha \in \mathbb{R},\lceil\alpha\rceil$ denotes the unique integer satisfying $\alpha \leq\lceil\alpha\rceil<\alpha+1$.

- For any $p \in \mathbb{N}$ we denote its bit-size by $\operatorname{bit}(p):=\left\lceil\log _{2}(p+1)\right\rceil$.

- For $(p, q) \in \mathbb{N}^{2}$, we set $\operatorname{bit}(p / q)=\operatorname{bit}(p)+\operatorname{bit}(q)$.

- For any tuplet of nonnegative integers $(a, b, c, M)$ we define

$$
\varphi(a, b, c, M):=a b(\operatorname{bit}(a)+\operatorname{bit}(b)+\operatorname{bit}(c)+\operatorname{bit}(M)) .
$$

In particular, for any $N \in \mathbb{N}$, one has $\varphi(n, d, N, 0)=n d(\operatorname{bit}(n)+\operatorname{bit}(d)+\operatorname{bit}(N))$.

We can now describe the classical model of stochastic games, as in Shapley [20].

Model. A stochastic game is described by a tuple $(K, I, J, g, q, k)$, where

- $K=\{1, \ldots, n\}$ is a finite set of states.

- For each $1 \leq \ell \leq n, I^{\ell}$ and $J^{\ell}$ are the sets of available actions for Player 1 and 2 , respectively, at state $\ell$.

- $g: Z \rightarrow \mathbb{R}$ is the reward function, where $Z:=\left\{(\ell, i, j) \mid \ell \in K,(i, j) \in I^{\ell} \times J^{\ell}\right\}$.

- $q: Z \rightarrow \Delta(K)$ is the transition function.

- $1 \leq k \leq n$ is the initial state.

The game proceeds in stages as follows. At each stage $m \geq 1$, both players are informed of the current state $k_{m} \in K$. Then, independently, Player 1 chooses an action $i_{m} \in I^{k_{m}}$ and Player 2 chooses an action $j_{m} \in J^{k_{m}}$. The pair $\left(i_{m}, j_{m}\right)$ is then observed by the players, from which they can infer the stage reward $g_{m}:=g\left(k_{m}, i_{m}, j_{m}\right)$. A new state $k_{m+1}$ is then chosen with the probability distribution $q\left(k_{m}, i_{m}, j_{m}\right)$, and the game proceeds to stage $m+1$. 
A $\lambda$-discounted stochastic game is one where Player 1 maximizes the expectation of $\sum_{m \geq 1} \lambda(1-\lambda)^{m-1} g_{m}$ while Player 2 minimizes the same amount, for some $\lambda \in(0,1]$.

An undiscounted stochastic game is one where Player 1 maximizes the expectation of $\liminf _{T \rightarrow+\infty} \frac{1}{T} \sum_{m=1}^{T} g_{m}$, while Player 2 minimizes the same amount.

Strategies. A (behavioral) strategy is a decision rule from the set of possible observations to the set of probabilities over the set of available actions. For every stage $m \geq 1$, the set of possible observations at stage $m$ is $Z^{m-1} \times K$. A strategy for Player 1 is thus a sequence of mappings $\sigma=\left(\sigma_{m}\right)_{m \geq 1}$ so that $\sigma_{m}\left(h_{m}, k_{m}\right) \in \Delta\left(I^{k_{m}}\right)$ for all $\left(h_{m}, k_{m}\right) \in Z^{m-1} \times K$. Similarly, a strategy for Player 2 is a sequence of mappings $\tau=\left(\tau_{m}\right)_{m \geq 1}$ so that $\tau_{m}\left(h_{m}, k_{m}\right) \in \Delta\left(J^{k_{m}}\right)$ for all $\left(h_{m}, k_{m}\right) \in Z^{m-1} \times K$. Both players choose their strategies independently. The sets of strategies are denoted, respectively, by $\Sigma$ and $\mathcal{T}$. By the Kolmogorov extension theorem, the initial state $k$, the transition function $q$, and a pair of strategies $(\sigma, \tau)$ induce a unique probability over the set of plays $Z^{\mathbb{N}}$, endowed with the sigma-algebra generated by the cylinders corresponding to finite histories, i.e. the sets $\left(z_{1}, \ldots, z_{p}\right) \times Z^{\mathbb{N}}$ for every $p \in \mathbb{N}$ and $\left(z_{1}, \ldots, z_{p}\right) \in Z^{p}$. This probability is denoted by $\mathrm{P}_{\sigma, \tau}^{k}$, and $\mathbb{E}_{\sigma, \tau}^{k}$ denotes the expectation with respect to $\mathrm{P}_{\sigma, \tau}^{k}$.

Stationary strategies. A stationary strategy is one that depends on the past observations only through the current state. A stationary strategy of Player 1, denoted by $x=\left(x^{1}, \ldots, x^{n}\right)$, is thus an element of $X$. Similarly, $y=\left(y^{1}, \ldots, y^{n}\right) \in Y$ is a stationary strategy of Player 2. The sets $I$ and $J$ are the sets of pure stationary strategies. We use the notation $\mathbf{i}=\left(\mathbf{i}^{1}, \ldots, \mathbf{i}^{n}\right) \in I$ and $\mathbf{j}=\left(\mathbf{j}^{1}, \ldots, \mathbf{j}^{n}\right) \in J$.

Discounted and undiscounted payoffs. To every pair $(\sigma, \tau) \in \Sigma \times \mathcal{T}$ corresponds a $\lambda$-discounted payoff for each discount rate $\lambda \in(0,1]$, and an undiscounted payoff, in the game $(K, I, J, g, q, k)$. They are given by

$$
\begin{aligned}
\gamma_{\lambda}^{k}(\sigma, \tau) & :=\mathbb{E}_{\sigma, \tau}^{k}\left[\sum_{m \geq 1} \lambda(1-\lambda)^{m-1} g_{m}\right], \\
\gamma^{k}(\sigma, \tau) & :=\mathbb{E}_{\sigma, \tau}^{k}\left[\liminf _{T \rightarrow+\infty} \frac{1}{T} \sum_{m=1}^{T} g_{m}\right] .
\end{aligned}
$$

The discounted values and the value. For each discount rate $\lambda \in(0,1]$, the $\lambda$-discounted stochastic game $(K, I, J, g, q, k)$ has a value, denoted by $v_{\lambda}^{k}$, whenever

$$
v_{\lambda}^{k}=\sup _{\sigma \in \Sigma} \inf _{\tau \in \mathcal{T}} \gamma_{\lambda}^{k}(\sigma, \tau)=\inf _{\tau \in \mathcal{T}} \sup _{\sigma \in \Sigma} \gamma_{\lambda}^{k}(\sigma, \tau)
$$

Similarly, the undiscounted stochastic game $(K, I, J, g, q, k)$ has a value, denoted by $v^{k}$, whenever

$$
v^{k}=\sup _{\sigma \in \Sigma} \inf _{\tau \in \mathcal{T}} \gamma^{k}(\sigma, \tau)=\inf _{\tau \in \mathcal{T}} \sup _{\sigma \in \Sigma} \gamma^{k}(\sigma, \tau)
$$


Classical results. The existence of $v_{\lambda}^{k}$ is due to Shapley [20], while Mertens and Neyman [16] proved the existence of $v^{k}$ and the equality $v^{k}=\lim _{\lambda \rightarrow 0} v_{\lambda}^{k}$.

In the sequel, we will refer to $v_{\lambda}^{k}$ and to $v^{k}$ as the $\lambda$-discounted value and the value, respectively, of the stochastic game $(K, I, J, g, q, k)$.

\subsection{Main results}

In the sequel, we consider stochastic games which can be described with rational data. For any $N \in \mathbb{N}$, we say that the stochastic game $(K, I, J, g, q, k)$ satisfies $\left(H_{N}\right)$ if $g(\ell, i, j)$ and $q\left(\ell^{\prime} \mid \ell, i, j\right)$ belong to the set $\left\{0, \frac{1}{N}, \frac{2}{N}, \ldots, 1\right\}$ for all $(\ell, i, j) \in Z$ and $1 \leq \ell^{\prime} \leq n$. Recall that $n=|K|$ is the number of states.

The main contributions of the present paper concern the computation of the discounted value $v_{\lambda}^{k}$ and the value $v^{k}$ of a stochastic game satisfying $\left(H_{N}\right)$. These numbers are known to be algebraic, that is there exists polynomials $P$ and $Q$ with integer coefficients and so that $P\left(v_{\lambda}^{k}\right)=0$ and $Q\left(v^{k}\right)=0$. For an algebraic number $\alpha \in \mathbb{R}$, an exact expression for $\alpha$ is a triplet $(P ; a, b)$ where $P$ is a polynomial with integer coefficients, $(a, b)$ is a pair of rational numbers, and $\alpha$ is the unique root of $P$ in the interval $(a, b)$. Thus, for instance, $\left(z^{2}-2 ; 1,2\right)$ is an exact expression for $\sqrt{2}$.

The complexity of the algorithms presented in this paper will be measured with the so-called logarithmic cost model which consists in assigning, to every arithmetic operation, a cost that is proportional to the number of bits involved. An algorithm is polynomial in the variables $t_{1}, \ldots, t_{m}$, if its logarithmic cost can be bounded by a polynomial expression of $t_{1}, \ldots, t_{m}$.

We can now state our results formally.

Theorem 1. There exists an algorithm that takes as input a stochastic game $(K, I, J, g, q, k)$ satisfying $\left(H_{N}\right)$ for some $N \in \mathbb{N}$ and a discount rate satisfying $\lambda \in\left\{0, \frac{1}{M}, \frac{2}{M}, \ldots, 1\right\}$ for some $M \in \mathbb{N}$, and outputs an exact expression for its discounted value $v_{\lambda}^{k}$. The algorithm is polynomial in $n,|I|,|J|, \log N$ and $\log M$.

Theorem 2. There exists an algorithm that takes as input a stochastic game $(K, I, J, g, q, k)$ satisfying $\left(H_{N}\right)$ for some $N \in \mathbb{N}$ and outputs an exact expression for its value $v^{k}$. The algorithm is polynomial in $n,|I|,|J|$ and $\log N$.

The algorithms that are mentioned in Theorems 1 and 2 are provided in Sections 4.2 and 4.3 respectively. Though very similar, the second algorithm has an additional

ingredient, namely a new bound on how small the discount rate needs to be so that $v_{\lambda}^{k}$ and $v^{k}$ are close to each other. This result, which has an interest in its own, can be formalized as follows. 
Theorem 3. For each $r \in \mathbb{N}$, set $\lambda_{r}:=2^{-4 n d(\operatorname{bit}(n)+\operatorname{bit}(d)+\operatorname{bit}(N))-r n d}$. Then, for any stochastic game $(K, I, J, g, q, k)$ satisfying $\left(H_{N}\right)$ one has

$$
\left|v_{\lambda}^{k}-v^{k}\right| \leq 2^{-r} \quad \forall \lambda \in\left(0, \lambda_{r}\right]
$$

Comments. The previous results deserve some comments. For simplicity, assume in these comments that $\left|I^{\ell}\right|=\left|J^{\ell}\right|=p$ for some $p \in \mathbb{N}$ and all $1 \leq \ell \leq n$.

1. $|I|=\prod_{\ell=1}^{n}\left|I^{\ell}\right|=p^{n}$ is the number of pure stationary strategies of Player 1 , and not the total number of actions $\sum_{\ell=1}^{n}\left|I^{\ell}\right|=n p$, and similarly for Player 2 .

2. Polynomial expressions in $|I|,|J|$ or $d$ are exponential expressions in $n$.

3. Theorems 1 and 2 improve the algorithms provided by Hansen et al. [10]. Our main achievement is two-fold: one the one hand, we reduce the dependence on $n$ from a double exponential to a simple exponential; on the other, our algorithms are considerably simpler and more direct.

4. Theorem 1 outperforms value iteration algorithms (such as the iteration of Shapley's operator) for small discount rates. Indeed, while our algorithm is polynomial in $p^{n}, n$ and $\log \lambda$, the latter is polynomial in $p, n$ and $\lambda$, for small $\lambda$.

5. For the $\lambda_{r}$ in Theorem 3 one has $\operatorname{bit}\left(\lambda_{r}\right)=O(n d(r+\operatorname{bit}(n)+\operatorname{bit}(d)+\operatorname{bit}(N))$, which is of order $p^{n}$. This result improves Proposition 22 of Hansen et al. [10], where an expression for the order of the bit-size of $\lambda_{r}$ is obtained in terms of big O's, namely, of order $p^{O\left(n^{2}\right)}$. Furthermore, the reduction from $p^{O\left(n^{2}\right)}$ to $p^{n}$ is fairly tight. Indeed, transposing Theorem 8 of [9] into the discounted case, it follows that one can construct an example for which a discount rate $\lambda$ of bit-size $p^{n / 2}$ is not enough to ensure that $v_{\lambda}^{k}$ and $v^{k}$ are close to each other.

\subsection{Selected past results}

We now gather some past results that will be used in our proofs. We start by defining the auxiliary matrices that were introduced in Attia and Oliu-Barton [1]. Consider the play induced by a pair $(\mathbf{i}, \mathbf{j}) \in I \times J$ of pure stationary strategies. Every time that the state $1 \leq \ell \leq n$ is reached, the players play $\left(\mathbf{i}^{\ell}, \mathbf{j}^{\ell}\right) \in I^{\ell} \times J^{\ell}$, so that the stage reward is $g\left(\ell, \mathbf{i}^{\ell}, \mathbf{j}^{\ell}\right)$ and the law of the next state is given by $q\left(\ell, \mathbf{i}^{\ell}, \mathbf{j}^{\ell}\right)$. Hence, the state variable follows a Markov chain with transition matrix $Q(\mathbf{i}, \mathbf{j}) \in \mathbb{R}^{n \times n}$ and the stage rewards can be described by a vector $g(\mathbf{i}, \mathbf{j}) \in \mathbb{R}^{n}$. For any $\lambda \in(0,1]$, let $\gamma_{\lambda}(\mathbf{i}, \mathbf{j}):=\left(\gamma_{\lambda}^{1}(\mathbf{i}, \mathbf{j}), \ldots \gamma_{\lambda}^{n}(\mathbf{i}, \mathbf{j})\right) \in \mathbb{R}^{n}$ be the vector of expected payoffs in the $\lambda$ discounted game, as the initial state varies from 1 to $n$. By stationarity, $Q(\mathbf{i}, \mathbf{j}), g(\mathbf{i}, \mathbf{j})$ and $\gamma_{\lambda}(\mathbf{i}, \mathbf{j})$ satisfy the recursive relation

$$
\gamma_{\lambda}(\mathbf{i}, \mathbf{j})=\lambda g(\mathbf{i}, \mathbf{j})+(1-\lambda) Q(\mathbf{i}, \mathbf{j}) \gamma_{\lambda}(\mathbf{i}, \mathbf{j})
$$

The matrix Id $-(1-\lambda) Q(\mathbf{i}, \mathbf{j})$ is invertible so that, by Cramer's rule, one has

$$
\gamma_{\lambda}^{k}(\mathbf{i}, \mathbf{j})=\frac{d_{\lambda}^{k}(\mathbf{i}, \mathbf{j})}{d_{\lambda}^{0}(\mathbf{i}, \mathbf{j})},
$$


where $d_{\lambda}^{0}(\mathbf{i}, \mathbf{j}):=\operatorname{det}(\operatorname{Id}-(1-\lambda) Q(\mathbf{i}, \mathbf{j})) \neq 0$ and where $d_{\lambda}^{k}(\mathbf{i}, \mathbf{j})$ is the determinant of the $n \times n$-matrix obtained by replacing the $k$-th column of $\operatorname{Id}-(1-\lambda) Q(\mathbf{i}, \mathbf{j})$ with $\lambda g(\mathbf{i}, \mathbf{j})$.

The auxiliary matrix of [1]. The auxiliary matrix $W_{\lambda}^{k}(z)$ is obtained by linearizing the quotient in (2.1) with an auxiliary variable $z \in \mathbb{R}$. Formally, for any $z \in \mathbb{R}$, one defines the $|I| \times|J|$ matrix $W_{\lambda}^{k}(z)$ by setting

$$
W_{\lambda}^{k}(z)[\mathbf{i}, \mathbf{j}]:=d_{\lambda}^{k}(\mathbf{i}, \mathbf{j})-z d_{\lambda}^{0}(\mathbf{i}, \mathbf{j}), \quad \forall(\mathbf{i}, \mathbf{j}) \in I \times J .
$$

Its value is denoted by val $W_{\lambda}^{k}(z)$.

The following two results are the main object of [1].

Theorem 2.1 For any $\lambda \in(0,1]$, $v_{\lambda}^{k}$ is the unique $z \in \mathbb{R}$ so that val $W_{\lambda}^{k}(z)=0$. Furthermore, the map $z \mapsto$ val $W_{\lambda}^{k}(z)$ is strictly decreasing.

Theorem $2.2 F^{k}(z):=\lim _{\lambda \rightarrow 0} \lambda^{-n}$ val $W_{\lambda}^{k}(z)$ exists in $\mathbb{R} \cup\{ \pm \infty\}$ for all $z \in \mathbb{R}$, and $v_{\lambda}^{k}$ converges, as $\lambda$ goes to 0 , to the unique $w \in \mathbb{R}$ so that $z>w \Rightarrow F^{k}(z)<0$ and $z<w \Rightarrow F^{k}(z)>0$. Furthermore, the map $z \mapsto F^{k}(z)$ is strictly decreasing.

The third result is contained in Theorem 2 of Shapley and Snow [21]. For any matrix $M$ of size $p \times p$, we denote by $S(M)$ the sum of the entries of the adjugate matrix of $M$, with the convention $S(M)=1$ if $p=1$ (i.e. when the adjugate matrix is not defined).

Theorem 2.3 For any matrix $M$ of size $p \times q$, there exists a square sub-matrix of $M$, denoted by $\dot{M}$, so that $S(\dot{M}) \neq 0$ and val $M=\frac{\operatorname{det} \dot{M}}{S(\dot{M})}$.

\section{Algebraic properties of the values}

Throughout this section, $(K, I, J, g, q, k)$ denotes a stochastic games satisfying $\left(H_{N}\right)$ for some $N \in \mathbb{N}$. Recall that a real number $\alpha$ is algebraic of degree $p$ if there exists a polynomial $P$ with integer coefficients satisfying $P(\alpha)=0$, and $p$ is the lowest degree of all such polynomials. The defining polynomial of $\alpha$ is the unique polynomial with integer coefficients $P(z)=a_{0}+a_{1} z+\cdots+a_{p} z^{p}$ so that $P(\alpha)=0, a_{p}>0$ and $\operatorname{gcd}\left(a_{0}, \ldots, a_{p}\right)=1$. In Section 3.1, we combine a technical result from Basu, Pollack and Roy [2] and Theorems 2.1, 2.2 and 2.3 to establish new bounds for the degree and the coefficients of the defining polynomials of $v_{\lambda}^{k}$ and $v^{k}$. These results will be used to analyze the algorithms corresponding to Theorems 1 and 2. In Section 3.2, we establish Theorem 3, a result that reduces the computation of the value of a stochastic game to the computation of its discounted value, for a well-chosen discount rate. This result will be used in the algorithm corresponding to Theorem 2 . 


\subsection{Bounds on the defining polynomials of the values}

We start by recalling Proposition 8.12 of Basu, Pollack and Roy [2].

Lemma 3.1 Let $A$ be an $p \times p$-matrix with polynomial entries in the variables $Y_{1}, \ldots, Y_{\ell}$ of degrees bounded by $q$ and integer coefficients of bit-size at most $\nu$. Then $\operatorname{det} A$, considered as a polynomial in $Y_{1}, \ldots, Y_{\ell}$ has degrees in $Y_{1}, \ldots, Y_{\ell}$ bounded by $p q_{1}, \ldots, p q_{\ell}$, and coefficients of bit-size at most $p \nu+p \operatorname{bit}(p)+\ell \operatorname{bit}(p q+1)$ where $q=\max \left(q_{1}, \ldots, q_{\ell}\right)$.

We can now use Lemma 3.1 and Theorem 2.3 to prove the following result.

Lemma 3.2 There exists two finite sets, denoted by $\mathcal{P}$ and $\mathcal{Q}$, which contain nonzero polynomials in the variables $(\lambda, z)$ of degree at most nd in $\lambda$ and $d$ in $z$ and integer coefficients, so that for each $(\lambda, z) \in(0,1] \times \mathbb{R}$, there exists $P \in \mathcal{P}$ and $Q \in \mathcal{Q}$ so that val $W_{\lambda}^{k}(z)=P(\lambda, z) / Q(\lambda, z), Q(\lambda, z) \neq 0$. Moreover, the coefficients of $P$ are of bit-size at most $3 \varphi(n, d, N, 0)$.

Proof. Let $(\mathbf{i}, \mathbf{j}) \in I \times J$ be fixed. By construction, $W_{\lambda}^{k}(z)[\mathbf{i}, \mathbf{j}]=d_{\lambda}^{k}(\mathbf{i}, \mathbf{j})-z d_{\lambda}^{0}(\mathbf{i}, \mathbf{j})$, where $d_{\lambda}^{k}(\mathbf{i}, \mathbf{j})$ and $d_{\lambda}^{0}(\mathbf{i}, \mathbf{j})$ are the determinants of two $n \times n$ matrices whose entries are polynomial in $\lambda$ of degree at most one and with coefficients in the set $\left\{0, \frac{1}{N}, \frac{2}{N}, \ldots, 1\right\}$. Consequently, $N^{n} W_{\lambda}^{k}(z)$ is a polynomial in $\lambda$ and $z$, of degree at most $n$ and 1 respectively, and integer coefficients whose bit-size is at most $\nu:=n \operatorname{bit}(n)+n \operatorname{bit}(N)+\operatorname{bit}(n+1)$ by Lemma 3.1. Let $\mathcal{P}$ and $\mathcal{Q}$ be, respectively, the sets of nonzero polynomials obtained as

$$
P(\lambda, z):=\operatorname{det}\left(N^{n} \dot{W}_{\lambda}^{k}(z)\right) \quad \text { and } \quad Q(\lambda, z):=S\left(N^{n} \dot{W}_{\lambda}^{k}(z)\right),
$$

when $\dot{W}_{\lambda}^{k}(z)$ ranges over all possible square sub-matrices of $W_{\lambda}^{k}(z)$. By Theorem 2.3, there exists a pair $(P, Q) \in \mathcal{P} \times \mathcal{Q}$ so that $Q(\lambda, z) \neq 0$ and

$$
\text { val } W_{\lambda}^{k}(z)=P(\lambda, z) /\left(N^{n} Q(\lambda, z)\right),
$$

where the normalization of the denominator is due to the fact that, for any square matrix $M$ of size $p \in \mathbb{N}$ and any $\alpha \in \mathbb{R}$, one has $\operatorname{det}(\alpha M)=\alpha^{p} \operatorname{det}(M)$ while $S(\alpha M)=$ $\alpha^{p-1} S(M)$. We now show that $P$ and $Q$ satisfy the desired properties. First, $P$ is nonzero as $z \mapsto$ val $W_{\lambda}^{k}(z)$ is strictly decreasing by Theorem 2.1. Second, the submatrices of $W_{\lambda}^{k}(z)$ are of size at most $d$ so that, by Lemma 3.1, all the polynomials in $\mathcal{P}$ and $\mathcal{Q}$ are of degree at most $n d$ in $\lambda$ and $d$ in $z$, and their coefficients are integers. From Lemma 3.1, one also obtains a bound for the bit-size of the coefficients of $P$, namely $d \nu+d \operatorname{bit}(d)+2 \operatorname{bit}(n d+1)$. Replacing $\nu$ in the last expression we an expression that be easily bounded by $3 \varphi(n, d, N, 0)$, which gives the desired result.

We are now ready to prove the main result of this section. Again, we assume in the sequel that $\lambda$ is a multiple of $1 / M$ for some $M \in \mathbb{N}$.

Proposition 3.3 The defining polynomials of $v_{\lambda}^{k}$ and $v^{k}$ are of degree at most $d$ and have coefficients of bit-size at most $4 \varphi(n, d, N, M)$ and $4 \varphi(n, d, N, 0)$, respectively. 
Proof. We start by proving the result for the discounted case. Let $\lambda$ be such that $\lambda M \in \mathbb{N}$. Let $P \in \mathcal{P}$ and $Q \in \mathcal{Q}$ be the two polynomials given in Lemma 3.2 for $z=v_{\lambda}^{k}$. Hence, $Q\left(\lambda, v_{\lambda}^{k}\right) \neq 0$ and val $W_{\lambda}^{k}\left(v_{\lambda}^{k}\right)=P\left(\lambda, v_{\lambda}^{k}\right) / Q\left(\lambda, v_{\lambda}^{k}\right)$. By Theorem 2.1, val $W_{\lambda}^{k}\left(v_{\lambda}^{k}\right)=0$, and consequently $P\left(\lambda, v_{\lambda}^{k}\right)=0$ by choice of $P$. Now, as $\lambda M \in \mathbb{N}$ and $P(\lambda, z)$ is a nonzero polynomial of degree at most $n d$ in $\lambda$ and $d$ in $z$ with integer coefficients, the following expression

$$
P_{\lambda}(z):=M^{n d} P(\lambda, z) \quad \forall z \in \mathbb{R},
$$

defines a nonzero polynomial of degree at most $d$ with integer coefficients and satisfying $P_{\lambda}\left(v_{\lambda}^{k}\right)=0$. Consequently, it is a multiple of the defining polynomial of $v_{\lambda}^{k}$. In particular, $v_{\lambda}^{k}$ has algebraic degree at most $d$. To bound the bit-size of the coefficients of $P_{\lambda}$ it is enough to note that $\operatorname{bit}\left(M^{n d} C\right) \leq n d \operatorname{bit}(M)+\operatorname{bit}(C)$ for any $C \in \mathbb{N}$, use the bound $3 \varphi(n, d, N, 0)$ obtained in Lemma 3.2 for $P$, and bound the bit-size of its factors with the Landau-Mignotte bound, which can be stated as follows (see Theorem 2 in [17]): for any three polynomials $A, B, C$ with integer coefficients, so that $A=\alpha_{0}+\alpha_{1} z \cdots+\alpha_{a} z^{a}$ and $B=\beta_{0}+\beta_{1} z+\cdots+\beta_{b} z^{b}$, and $A=B C$, one has $\max \left(\left|\beta_{0}\right|, \ldots,\left|\beta_{b}\right|\right) \leq\left(\begin{array}{c}b \\ \ulcorner b / 2\rceil\end{array}\right)\left(\alpha_{0}^{2}+\cdots+\alpha_{a}^{2}\right)^{1 / 2}$. The Landau-Mignotte bound thus adds an additional term $(d+1) \operatorname{bit}(d)$ to the previous bound. The result follows then from the relation $3 \varphi(n, d, N, 0)+(d+1) \operatorname{bit}(d) \leq 4 \varphi(n, d, N, 0)$.

Consider now the undiscounted case. As already argued in the discounted case, for each $\lambda \in(0,1]$ there exists a nonzero polynomial $P \in \mathcal{P}$ of degree at most $n d$ in $\lambda$ and $d$ in $z$ (the choice of the polynomial depends on $\lambda$ ), with integer coefficients of bit-size at most $3 \varphi(n, d, N, 0)$, and so that $P\left(\lambda, v_{\lambda}^{k}\right)=0$. By finiteness of the set $\mathcal{P}$, and because two polynomials cannot intersect infinitely many times in $(0,1]$, one of these polynomials must satisfy $P\left(\lambda, v_{\lambda}^{k}\right)=0$ for all $\lambda$ sufficiently small. For this polynomial, denoted again by $P(\lambda, z)$, let $P_{0}, \ldots, P_{n d}$ be the unique polynomials in $z$ so that

$$
P(\lambda, z)=P_{0}+\lambda P_{1}(z)+\cdots+\lambda^{n d} P_{n d}(z)=0 .
$$

As $P(\lambda, z)$ is nonzero, there exists $0 \leq s \leq n d$ and $P_{s} \neq 0$ so that

$$
P(\lambda, z)=\lambda^{s} P_{s}(z)+o\left(\lambda^{s}\right)
$$

By construction, $P_{s}$ is a nonzero polynomial of degree at most $d$ and has integer coefficients of bit-size at most $3 \varphi(n, d, N, 0)$. Dividing by $\lambda^{s}$, and letting $\lambda$ go to 0 ,

$$
0=\lim _{\lambda \rightarrow 0} \frac{P\left(\lambda, v_{\lambda}^{k}\right)}{\lambda^{s}}=P_{s}\left(v^{k}\right) .
$$

Hence, $P_{s}$ is a multiple of the defining polynomial of $v^{k}$. Like in the discounted case, we obtain the desired bound from the Landau-Mignotte bound.

Comments. This result, which relies on Theorems 2.1, 2.2 and 2.3, improves the bound provided by Hansen et al. [10]. To see this, consider the case where $\left|I^{\ell}\right|=$ $\left|J^{\ell}\right|=p$ for some $p \in \mathbb{N}$ and all $1 \leq \ell \leq n$ and $\lambda N \in \mathbb{N}$. In this case, [10] bounded the algebraic degree of $v_{\lambda}^{k}$ and $v^{k}$ by $(2 p+5)^{n}$, while Proposition 3.3 reduces the bound to $d=p^{n}$. Furthermore, this bound is tight. Our result also reduces the bound on the bit-size of the coefficients obtained therein, from $22 p^{2} n^{2}(2 p+5)^{n} \operatorname{bit}(N)$ to $4 n p^{n}(\operatorname{bit}(n)+\operatorname{bit}(p)+\operatorname{bit}(N))$. 


\subsection{The distance between $v_{\lambda}^{k}$ and $v^{k}$}

In this section we establish Theorem 3. First of all, recall the following classical bounds from Cauchy [6] and Mahler [15] concerning the roots of polynomial.

Lemma 3.4 Let $P(z)=a_{0}+a_{1} z \cdots+a_{p} z^{\ell}$ be a non zero polynomial with integer coefficients, and let $\|P\|_{\infty}:=\max \left(\left|a_{0}\right|, \ldots,\left|a_{p}\right|\right)$ and $\|P\|_{2}:=\left(\sum_{r=0}^{p} a_{r}^{2}\right)^{1 / 2}$. Then,

(i) If $\alpha$ is a root of $P$ then $\alpha \geq \frac{1}{2\|P\|_{\infty}}$.

(ii) If $\beta \neq \alpha$ is another root of $P$ then $|\alpha-\beta| \geq p^{-(p+2) / 2}\|P\|_{2}^{1-p}$.

The following result is a direct consequence of Proposition 3.3 and Lemma 3.4 (ii).

Lemma 3.5 Let $P$ be the defining polynomial of $v_{\lambda}^{k}$, and let $\varepsilon \leq 2^{-8 d \varphi(n, d, N, M)}$. Then $P$ has no root in the interval $\left(v_{\lambda}^{k}-\varepsilon, v_{\lambda}^{k}+\varepsilon\right)$. Similarly, let $Q$ be the defining polynomial of $v^{k}$ and let $\varepsilon \leq 2^{-8 d \varphi(n, d, N, 0)}$. Then $Q$ has no root in the interval $\left(v^{k}-\varepsilon, v^{k}+\varepsilon\right)$.

Proof. Let us start by $v_{\lambda}^{k}$. By definition of the defining polynomial $P\left(v_{\lambda}^{k}\right)=0$. By Proposition 3.3, $P$ is of degree at most $d$ and its integer coefficients are bounded by $C:=2^{4 \varphi(n, d, N, M)}$. Consequently, $\|P\|_{2}^{2} \leq C^{2}(d+1)$ and, by Lemma $3.4(i i)$, any other root $z$ of $P$ satisfies

$$
\begin{aligned}
\left|z-v_{\lambda}^{k}\right| & \geq d^{-(d+2) / 2}(d+1)^{(1-d) / 2} C^{1-d} \\
& \geq 2^{-8 d \varphi(n, d, N, M)}
\end{aligned}
$$

This inequality proves the statement for $v_{\lambda}^{k}$. We omit the proof for $v^{k}$ as it goes along the exact same lines: it is enough to replace $P, v_{\lambda}^{k}$ and $\varphi(n, d, N, M)$ with $Q, v^{k}$ and $\varphi(n, d, N, 0)$.

Using Lemma $3.4(i)$, Lemma 3.2, and Theorem 2.3, we now derive some valuable insight on the asymptotic behavior of the sign of the map $\lambda \mapsto \operatorname{val} W_{\lambda}^{k}(z)$ as $\lambda$ goes to 0 , for a well-chosen fixed $z \in \mathbb{R}$. This result will be crucial in the proof of Theorem 3 .

Proposition 3.6 For any $r \in \mathbb{N}$, set $Z_{r}:=\left\{0, \frac{1}{2^{r}}, \ldots, \frac{2^{r}}{2^{r}}\right\}$ and $\lambda_{r}:=2^{-4 \varphi(n, d, N, 0)-r n d}$. Then, for each $z \in Z_{r}$,

$$
\left\{\begin{array}{l}
\operatorname{val} W_{\lambda_{r}}^{k}(z)>0 \Longrightarrow F^{k}(z) \in[0,+\infty] \\
\operatorname{val} W_{\lambda_{r}}^{k}(z)<0 \Longrightarrow F^{k}(z) \in[-\infty, 0] \\
\operatorname{val} W_{\lambda_{r}}^{k}(z)=0 \Longrightarrow F^{k}(z)=0 .
\end{array}\right.
$$

Proof. Let $z \in Z_{r}$ be fixed. Let $\mathcal{P}$ and $\mathcal{Q}$ be the set of polynomials of Lemma 3.2. Hence, for all $P \in \mathcal{P}$, the polynomial $P(\lambda, z)$ is of degree at most $n d$ in $\lambda$ and $d$ in $z$. Furthermore, by the choice of $z$,

$$
P_{z}(\lambda):=2^{r n d} P(\lambda, z) \quad \lambda \in(0,1]
$$

defines a polynomial in the variable $\lambda$ of degree at most $n d$ and with integer coefficients of bit-size at most $3 \varphi(n, d, N, 0)+r n d+1$. Let $\mathcal{P}(z)$ and $\mathcal{Q}(z)$ be the set of all the polynomials obtained this way, as $P$ and $Q$ range, respectively, in the sets $\mathcal{P}$ and $\mathcal{Q}$. 
By Theorem 2.3, for any $\lambda \in(0,1]$ there exists $P_{z} \in \mathcal{P}(z)$ and $Q_{z} \in \mathcal{Q}(z)$, the choice of the polynomials depends on $\lambda$, so that

$$
\operatorname{val} W_{\lambda}^{k}(z)=\frac{P_{z}(\lambda)}{Q_{z}(\lambda)}
$$

Hence, a necessary condition for the function $\lambda \mapsto$ val $W_{\lambda}^{k}(z)$ to change sign at some $\alpha \in \mathbb{R}$ is that $P_{z}(\alpha)=0$ for some polynomial $P_{z} \in \mathcal{P}(z)$. Applying Lemma $3.4(i)$ to the nonzero polynomials in $\mathcal{P}(z)$, it follows that neither of them admits a root in the interval $\left(0, \lambda_{r}\right]$. In other words, the sign of $\lambda \mapsto$ val $W_{\lambda}^{k}(z)$ is constant in the interval $\left(0, \lambda_{r}\right]$. Consider now the three possible cases, val $W_{\lambda_{r}}^{k}(z)>0$, val $W_{\lambda_{r}}^{k}(z)<0$, and val $W_{\lambda_{r}}^{k}(z)=0$. In the first case, $\lambda^{-n}$ val $W_{\lambda}^{k}(z)>0$ for all $\lambda \in\left(0, \lambda_{r}\right]$ so that

$$
F^{k}(z):=\lim _{\lambda \rightarrow 0} \lambda^{-n} \operatorname{val} W_{\lambda}^{k}(z) \in[0,+\infty]
$$

The second case is similar. For the third, val $W_{\lambda_{r}}^{k}(z)=0$ implies that val $W_{\lambda}^{k}(z)=0$ for all $\lambda \in\left(0, \lambda_{r}\right]$ so that one also has $F^{k}(z)=0$.

We are now ready to establish Theorem 3, whose statement is as follows. For each $r \in \mathbb{N}$, let $\lambda_{r}:=2^{-4 \varphi(n, d, N, 0)-r n d}$. Then $\left|v_{\lambda}^{k}-v^{k}\right| \leq 2^{-r}$ for all $\lambda \in\left(0, \lambda_{r}\right)$.

Proof of Theorem 3. Let $\lambda \in\left(0, \lambda_{r}\right]$ be fixed. First of all, the maps $z \mapsto$ val $W_{\lambda}^{k}(z)$ and $z \mapsto F^{k}(z)$ are strictly decreasing, by Theorems 2.1 and 2.2, respectively. Therefore, either there exists a unique $z \in Z_{r}=\left\{0, \frac{1}{2^{r}}, \ldots, \frac{2^{r}}{2^{r}}\right\}$ so that val $W_{\lambda}^{k}(z)=0$, or there exists $0 \leq m \leq 2^{r}$ such that val $W_{\lambda}^{k}\left(m 2^{-r}\right)>0$ and val $W_{\lambda}^{k}\left((m+1) 2^{-r}\right)<0$, and the same is true for $F^{k}$. Consider the first case, and let $z \in Z_{r}$ satisfy val $W_{\lambda}^{k}(z)=$ 0 . By Theorem 2.1, this implies $z=v_{\lambda}^{k}$, so that, by Proposition 3.6, one also has $F^{k}(z)=0$. But then, Theorem 2.2 implies $v^{k}=z$ so that $v_{\lambda}^{k}=v^{k}$, and the inequality $\left|v_{\lambda}^{k}-v^{k}\right| \leq 2^{-r}$ holds. Consider now the second case, and let $1 \leq m \leq 2^{r}$ be such that val $W_{\lambda}^{k}\left(m 2^{-r}\right)>0$ and val $W_{\lambda}^{k}\left((m+1) 2^{-r}\right)<0$. On the one hand, Theorem 2.1 implies

$$
m 2^{-r}<v_{\lambda}^{k}<(m+1) 2^{-r} .
$$

On the other, Proposition 3.6 gives $F^{k}\left(m 2^{-r}\right) \geq 0$ and $F^{k}\left((m+1) 2^{-r}\right) \leq 0$ which, in view of Theorem 2.2, implies

$$
m 2^{-r} \leq v^{k} \leq(m+1) 2^{-r} .
$$

The combination of (3.1) and (3.2) yields the desired inequality $\left|v_{\lambda}^{k}-v^{k}\right| \leq 2^{-r}$.

\section{Algorithms}

The aim of this section is to describe the algorithms that correspond to Theorems 1 and 2. We start by recalling three classical algorithms in Section 4.1 that are called by the above-mentioned algorithms. Section 4.2 is devoted to the description of two algorithms: the first one outputs arbitrarily close approximations for the discounted 
values of a stochastic game, while the second one outputs an exact expression for this value. The latter corresponds to the algorithm of Theorem 1. Similarly, Section 4.2 is devoted to the description of three algorithms: the first two output arbitrarily close approximations for the value of a stochastic game, while the third one outputs anexact expression for this value. The latter corresponds to the algorithm of Theorem 2 .

\subsection{Auxiliary results}

Recall that the complexity of the algorithms is measured with the logarithmic cost model. The logarithmic cost of an algorithm can be bounded by 1) a bound of the number of arithmetic operations that it requires, and 2) a bound the bit-size of the numbers that are involved in them. In particular, if these two bounds are polynomial expressions in some variables $t_{1}, \ldots, t_{m}$, so is the logarithmic cost of the algorithm.

We now recall three well-known algorithms. The first one, due to Kannan, Lenstra and Lovász [12], allows to compute the defining polynomial of an algebraic number efficiently. It will be referred as the KLL algorithm. The second, due to Karmarkar [13], allows to solve linear programs efficiently and will be referred to as the Karmarkar algorithm. The third one, which allows to compute the determinant of a square matrix efficiently, is taken from Basu, Pollack and Roy [2], where it is referred to as the Dodgson-Jordan-Bareiss algorithm.

Theorem 4.1 Let $\alpha \in \mathbb{R}$ be an algebraic number of degree $p \in \mathbb{N}$ and defining polynomial $P(z)=a_{0}+a_{1} z+\cdots+a_{p} z^{p}, z \in \mathbb{R}$. The KLL algorithm outputs the defining polynomial of $\alpha \in \mathbb{R}$ when given as inputs $(D, C, \bar{\alpha})$ satisfying

- $B \in \mathbb{N}$ is a bound on the algebraic degree of $\alpha$, i.e. $p \leq D$.

- $2^{C} \in \mathbb{N}$ bounds the integer coefficients $\left|a_{0}\right|, \ldots,\left|a_{p}\right|$.

- $\bar{\alpha} \in \mathbb{Q}$ so that $|\alpha-\bar{\alpha}| \leq 2^{-s} \frac{1}{12 D}$, where

$$
s=s(b, C):=\left\lceil D^{2} / 2+(3 D+4) \log _{2}(D+1)+2 D C\right\rceil .
$$

This algorithm requires $O\left(p D^{4}(D+C)\right)$ arithmetic operations on integers of bit-size $O\left(D^{2}(D+C)\right)$.

Theorem 4.2 Let $M$ be a $p \times q$ matrix with rational entries which can be encoded in $C$ bits. The Karmarkar algorithm inputs $M$ and outputs its value val $M$, and requires $O\left(p^{3.5} C\right)$ arithmetic operations on integers of bit-size at most $O(C)$.

Theorem 4.3 Let $M$ be a $p \times p$ matrix with integer entries of bit-size $C$. The DodgsonJordan-Bareiss algorithm inputs $M$ and outputs $\operatorname{det} M$, and requires $O\left(p^{3}\right)$ arithmetic operations on integers of bit-size $O(p \operatorname{bit}(p) C)$.

For the three above-mentioned algorithms, the following assertions hold.

- The KLL algorithm is polynomial in $D$ and $C$, the bounds for the degree and the bit-size of the coefficients, respectively, of the defining polynomial of $\alpha$.

- The Karmarkar algorithm and the Dogson-Jordan-Bareiss algorithm are polynomial in the size of the matrix and in the bit-size of its entries. 


\subsection{Computing the discounted values}

We start by describing a bisection algorithm, directly derived from Theorem 2.1, that outputs arbitrarily close approximations of the $\lambda$-discounted value $v_{\lambda}^{k}$ of a stochastic game $(K, I, J, g, q, k)$. As we will show later on (see Algorithm 2 approx bis), this algorithm can also be used to obtain arbitrarily close approximations of $v^{k}$, thanks to Theorem 3.

\section{Algorithm 1 approx}

Input: A stochastic game $(K, I, J, g, q, k)$ satisfying $\left(H_{N}\right)$ for some $N \in \mathbb{N}$, a discount rate $\lambda$ satisfying $\lambda M \in \mathbb{N}$ for some $M \in \mathbb{N}$, and a precision level $r \in \mathbb{N}$.

Output: A rational number $u \in\left\{0, \frac{1}{2^{r}}, \ldots, \frac{2^{r}-1}{2^{r}}\right\}$ so that $v_{\lambda}^{k} \in\left[u, u+\frac{1}{2^{r}}\right]$.

Computation cost: Polynomial in $n,|I|,|J|, \log N, \log M$ and $r$.

1. Set $\underline{w}:=0, \bar{w}:=1$

2. WHILE $\bar{w}-\underline{w}>2^{-r}$ DO

$2.1 z:=\frac{\underline{w}+\bar{w}}{2}$

2.2 Compute $W_{\lambda}^{k}(z)$

2.3 Compute $v:=\operatorname{val} W_{\lambda}^{k}(z)$

2.4 IF $v \geq 0$, THEN $\underline{w}:=z$

2.5 IF $v \leq 0$ THEN $\bar{w}:=z$

3. RETURN $u:=\underline{w}$.

Computation cost of Algorithm 1 approx. By Theorem 2.1, each iteration of Step 2 reduces the interval $[\underline{w}, \bar{w}]$ by a factor of $1 / 2$, while satisfying $\underline{w} \leq v_{\lambda}^{k} \leq \bar{w}$. Consequently, the algorithm terminates after at most $r$ steps, and the output $u$ satisfies $v_{\lambda}^{k} \in\left[u, u+\frac{1}{2^{r}}\right]$. As Steps 2.1, 2.4 and 2.5 require one operation each, the computation cost of the algorithm depends essentially on the computation cost of Steps 2.2 and 2.3, which is the object of the following lemma.

Lemma 4.4 Let $L=O(n \operatorname{bit}(n) \operatorname{bit}(N) \operatorname{bit}(M))$. For all $r \in \mathbb{N}$ and $z \in\left\{0, \frac{1}{2^{r}}, \ldots, \frac{2^{r}-1}{2^{r}}\right\}$,

(i) The computation of $W_{\lambda}^{k}(z)$ with the Dogson-Jordan-Bareiss algorithm requires $O\left(n^{3}|I||J|\right)$ arithmetic operations, with numbers of bit-size $O(L+r)$.

(ii) The computation of val $W_{\lambda}^{k}(z)$ with the Karmarkar algorithm requires $O\left(d^{3.5}(L+\right.$ $r)$ ) arithmetic operations, with numbers of bit-size $O(L+r)$.

Proof. Let $r \in \mathbb{N}$ and $z \in\left\{0, \frac{1}{2^{r}}, \ldots, \frac{2^{r}-1}{2^{r}}\right\}$ be fixed.

(i) Thanks to the assumptions $\left(H_{N}\right)$ and $\lambda M \in \mathbb{N}$, and by the definition of $W_{\lambda}^{k}(z)$, for each $(\mathbf{i}, \mathbf{j}) \in I \times J, W_{\lambda}^{k}(z)[\mathbf{i}, \mathbf{j}]=d_{\lambda}^{0}(\mathbf{i}, \mathbf{j})-z d_{\lambda}^{0}(\mathbf{i}, \mathbf{j})$ where $d_{\lambda}^{0}(\mathbf{i}, \mathbf{j})$ and $d_{\lambda}^{k}(\mathbf{i}, \mathbf{j})$ are 
determinants of some $n \times n$ matrices whise entries are multiples of $\frac{1}{N M}$. Multiplying each entry by $N M$, so that all entries are integers, it follows then from Theorem 4.3 that $d_{\lambda}^{0}(\mathbf{i}, \mathbf{j})$ and $d_{\lambda}^{0}(\mathbf{i}, \mathbf{j})$ can be computed in $O\left(n^{3}\right)$ arithmetic operations on integers of bit-size $O(n \operatorname{bit}(n) \operatorname{bit}(N M))=O(L)$. The entries of $W_{\lambda}^{k}(z)$ are then of bit-size at most $O(L+r)$ because, by the choice of $z$, bit $(z)=O(r)$. Finally, the total number of operations is simply $O\left(n^{3}|I||J|\right)$ because the matrix $W_{\lambda}^{k}(z)$ is of size $|I| \times|J|$.

(ii) As already noted in the poof of $(i)$, the entries $W_{\lambda}^{k}(z)$ are of bit-size at most $O(L+r)$. The result follows then directly from Theorem 4.2.

The next result is a direct consequence from Lemma 4.4.

Theorem 4.5 Algorithm 1 approx computes a $2^{-r}$-approximation of $v_{\lambda}^{k}$ for any $r \in \mathbb{N}$, and its computation cost is polynomial in $n,|I|,|J|, \log N, \log M$ and $r$.

Next, we combine Algorithm 1 approx and the KLL algorithm in order to obtain an exact expression for $v_{\lambda}^{k}$.

\section{Algorithm 1 exact}

Input: A stochastic game $(K, I, J, g, q, k)$ satisfying $\left(H_{N}\right)$ for some $N \in \mathbb{N}$, and a discount rate $\lambda \in(0,1]$ so that $\lambda M \in \mathbb{N}$ for some $M \in \mathbb{N}$.

Output: An exact expression for $v_{\lambda}^{k}$.

Computation cost: Polynomial in $n,|I|,|J|, \log N$ and $\log M$.

1. Initialization phase

1.1 Set $C:=4 \varphi(n, d, N, M)$

1.2 Set $s:=\left\lceil d^{2} / 2+(3 d+4) \log _{2}(d+1)+2 d C\right\rceil$

1.3 Set $r:=s\left\lceil\log _{2} 12 d\right\rceil$

2. Run Algorithm 1 approx with inputs $(K, I, J, g, q, k)$, the discount rate $\lambda$, and a precision level $r$. Denote its output by $u$.

3. Run the $K L L$ algorithm with inputs $d, C$ and $u$, and output $P$.

4. RETURN $\left(P ; u, u+2^{-r}\right)$.

We are now ready to prove Theorem 1. More precisely, we show that Algorithm 1 exact computes an exact expression for $v_{\lambda}^{k}$, and that (its computation cost) is polynomial in $n,|I|,|J|, \log N$ and $\log M$. 
Proof of Theorem 1. First, recall that the algebraic degree of $v_{\lambda}^{k}$, and the bit-size of the coefficients of its defining polynomial, are bounded by $d$ and $C$ respectively, by Proposition 3.3. Second, by Theorem 4.5, Step 2 of Algorithm 1 exact returns $u$ so that $\operatorname{bit}(u) \leq 2 r$ and $\left|u-v_{\lambda}^{k}\right| \leq 2^{-r}$, and the computation cost is polynomial in $n,|I|$, $|J|, \log N, \log M$ and $r$. Third, by Theorem 4.1, the definition of $C, r$ and $s$ in Step 1 of Algorithm 1 exact ensure that Step 3 of Algorithm 1 exact provides the defining polynomial $P$ of $v_{\lambda}^{k}$, and that the computation cost is polynomial in $d$ and $C$. As $C$ and $r$ are (bounded by) polynomial expressions in $n, d$ and $\log N$, the entire algorithm is thus polynomial in $n,|I|,|J|, \log N$ and $\log M$. It remains to show that $P$ has no other root than $v_{\lambda}^{k}$ in the interval $\left(u, u+2^{-r}\right)$ so that $\left(P ; u, u+2^{-r}\right)$ is an exact expression for $v_{\lambda}^{k}$. To see this, note that by definition one has $r \geq 8 d \varphi(n, d, N, M)$. By Lemma 3.5 , this implies that $P$ has no other root in the interval $\left(v_{\lambda}^{k}-2^{-r}, v_{\lambda}^{k}+2^{-r}\right)$, and the result follows because this interval contains $\left(u, u+2^{-r}\right)$ thanks to $\left|u-v_{\lambda}^{k}\right| \leq 2^{-r}$.

\subsection{Computing the value}

Like for the discounted case, we start by proposing a bisection algorithm, directly derived from Theorem 2.2, which outputs arbitrarily close approximations of the value $v^{k}$ of a stochastic game $(K, I, J, g, q, k)$. Note, however, that the natural algorithm would consist in computing the $\operatorname{sign}$ of $F^{k}(z):=\lim _{\lambda \rightarrow 0} \lambda^{-n}$ val $W_{\lambda}^{k}(z)$ at each iteration, but this computation seems very costly. Luckily, there is a way out to this issue. Indeed, by Proposition 3.6, this computation is equivalent to that of the sign of val $W_{\lambda}^{k}(z)$, for a well-chosen $\lambda$, and this can be done efficiently because it is a linear program (provided that the bit-size of $\lambda$ is polynomial). The following bisection algorithm is built upon this observation.

\section{Algorithm 2 approx}

Input: A stochastic game $(K, I, J, g, q, k)$ satisfying $\left(H_{N}\right)$ for some $N \in \mathbb{N}$, and a precision level $r \in \mathbb{N}$.

Output: A rational number $u \in\left\{0, \frac{1}{2^{r}}, \ldots, \frac{2^{r}-1}{2^{r}}\right\}$ so that $v^{k} \in\left[u, u+\frac{1}{2^{r}}\right]$.

Computation cost: Polynomial in $n,|I|,|J|, \log N$ and $r$.

1.1. Set $\lambda_{r}:=2^{-4 \varphi(n, d, N, 0)-r n d}$

1.2. Set $\underline{w}:=0, \bar{w}:=1$

2. WHILE $\bar{w}-\underline{w}>2^{-r}$ DO

$2.1 z:=\frac{\underline{w}+\bar{w}}{2}$

2.2 Compute $W_{\lambda_{r}}^{k}(z)$

2.3 Compute $v:=\operatorname{val} W_{\lambda_{r}}^{k}(z)$

2.4 IF $v \geq 0$, THEN $\underline{w}:=z$

2.5 IF $v \leq 0$ THEN $\bar{w}:=z$ 
The next result is a direct consequence of Proposition 3.6, Lemma 4.4 and the definition of $\lambda_{r}$.

Theorem 4.6 Algorithm 2 approx computes a $2^{-r}$-approximation of $v^{k}$ for any $r \in \mathbb{N}$, and its computation cost is polynomial in $n,|I|,|J|, \log N$ and $r$.

Proof. By Proposition 3.6, the sign of $W_{\lambda_{r}}^{k}(z)$ coincides with the sign of $F^{k}(z)=$ $\lim _{\lambda \rightarrow 0} \lambda^{-n}$ val $W_{\lambda}^{k}(z)$ at every $z$ that is called by the algorithm. It follows then from Theorem 2.2 that Algorithm 2 approx provides a $2^{-r}$-approximation of $v^{k}$. By Lemma 4.4, its computation cost is polynomial in $n, d$, $\operatorname{bit}(N)$ and $\operatorname{bit}\left(\lambda_{r}\right)$. The result follows then from the fact that the bit-size of $\lambda_{r}$ is polynomial in $n, d, \log N$ and $r$.

Alternatively, one can use Theorem 3 to obtain arbitrary close approximation for $v^{k}$ directly from Algorithm 1 approx, as follows.

\section{Algorithm 2 approx bis}

Input: A stochastic game $(K, I, J, g, q, k)$ satisfying $\left(H_{N}\right)$ for some $N \in \mathbb{N}$, and a precision level $r \in \mathbb{N}$.

Output: A rational number $u \in\left\{0, \frac{1}{2^{r}}, \ldots, \frac{2^{r}-1}{2^{r}}\right\}$ so that $v^{k} \in\left[u, u+\frac{1}{2^{r}}\right]$.

Computation cost: Polynomial in $n,|I|,|J|, \log N$ and $r$.

1. Set $\lambda_{r+1}:=2^{-4 \varphi(n, d, N, 0)-(r+1) n d}$.

2. Run Algorithm 1 approx with inputs the stochastic game $(K, I, J, g, q, k)$, the discount rate $\lambda_{r+1}$ and the precision level $r+1$. Let $u$ denote its output.

3. RETURN $u$.

Theorem 4.7 Algorithm 2 approx bis computes a $2^{-r}$-approximation of $v^{k}$ for any $r \in \mathbb{N}$, and its computation cost is polynomial in $n,|I|,|J|, \log N$ and $r$.

Proof. By Theorem 4.5, Step 2 of Algorithm 2 approx bis outputs $u$ so that $\mid u-$ $v_{\lambda_{r+1}}^{k} \mid \leq \frac{1}{2^{r+1}}$, and the cost is polynomial in $n,|I|,|J|, \log N, r$ and $\operatorname{bit}\left(\lambda_{r}\right)$. The latter being polynomial $n,|I|,|J|, \log N$ and $r$, the cost is thus polynomial in these variables too. Finally, by the choice of $\lambda_{r+1}$, Theorem 3 implies $\left|v^{k}-v_{\lambda_{r+1}}^{k}\right| \leq \frac{1}{2^{r+1}}$. The result follows, since

$$
\left|u-v^{k}\right| \leq\left|u-v_{\lambda_{r+1}}^{k}\right|+\left|v^{k}-v_{\lambda_{r+1}}^{k}\right| \leq \frac{1}{2^{r}}
$$


Like in the discounted case, one can now combine Algorithm 2 approx (or Algorithm 2 approx bis) with the KLL algorithm to obtain an algorithm that outputs an exact expression for $v^{k}$. The algorithm goes as follows.

Algorithm 2 exact

Input: A finite stochastic game $(K, I, J, g, q, k)$ satisfying $\left(H_{N}\right)$ for some $N \in \mathbb{N}$.

Output: An exact expression for $v^{k}$.

Computation cost: Polynomial in $n,|I|,|J|$ and $\log N$.

1. Initialization phase.

1.1 Set $C:=4 \varphi(n, d, N, 0)$.

1.2 Set $s:=\left\lceil d^{2} / 2+(3 d+4) \log _{2}(d+1)+2 d C\right\rceil$.

1.3 Set $r:=s\left\lceil\log _{2} 12 d\right\rceil$.

2. Run Algorithm 2 approx bis with inputs the stochastic game $(K, I, J, g, q, k)$ and the precision level $r+1$. Denote its output by $u$.

3. Run the $K K L$ algorithm with inputs $d, C$ and $u$. Denote its output by $Q$.

4. $\operatorname{RETURN}\left(Q ; u, u+2^{-r}\right)$.

We are now ready to prove Theorem 1. More precisely, we show that Algorithm 2 exact computes an exact expression for $v^{k}$, and that (its computation cost) is polynomial in $n,|I|,|J|$ and $\log N$. The proof is similar to that of Theorem 1 .

Proof of Theorem 2. First, recall that the algebraic degree of $v^{k}$, and the bit-size of the coefficients of its defining polynomial, are bounded by $d$ and $C$ respectively, by Proposition 3.3. Second, by Theorem 4.7, Step 2 of Algorithm 2 exact returns $u$ so that $\operatorname{bit}(u) \leq 2 r$ and $\left|u-v^{k}\right| \leq 2^{-r}$, and the computation cost is polynomial in $n$, $|I|,|J|, \log N$, and $r$. Third, by Theorem 4.1, the definition of $C, r$ and $s$ in Step 1 of Algorithm 1 exact ensure that Step 3 of Algorithm 2 exact provides the defining polynomial $Q$ of $v^{k}$, and that the computation cost is polynomial in $d$ and $C$. As $C$ and $r$ are (bounded by) polynomial expressions in $n, d$ and $\log N$, the entire algorithm is thus polynomial in $n,|I|,|J|$ and $\log N$. It remains to show that $Q$ has no other root than $v^{k}$ in the interval $\left(u, u+2^{-r}\right)$ so that $\left(Q ; u, u+2^{-r}\right)$ is an exact expression for $v^{k}$. To see this, note that by definition one has $r \geq 8 d \varphi(n, d, N, 0)$. By Lemma 3.5 , this implies that $Q$ has no other root in the interval $\left(v^{k}-2^{-r}, v^{k}+2^{-r}\right)$, and the result follows because this interval contains $\left(u, u+2^{-r}\right)$ thanks to $\left|u-v^{k}\right| \leq 2^{-r}$.

\subsection{An example}

We now provide an example to illustrate our results. The stochastic game goes back to Kohlberg [4]. It is played over 4 states, two of which are absorbing, i.e. once 
these states are reached, the state can no longer change. The game can be described as follows, where each box represents a state, arrows indicate deterministic transitions from the current state to the state that is pointed, and numbers indicate stage rewards:

\begin{tabular}{|c|c|}
\hline $1 \circlearrowleft$ & $0 \rightarrow$ \\
\hline $0 \rightarrow$ & $0 \downarrow$ \\
\hline
\end{tabular}

\begin{tabular}{|c|c|c|}
\hline-1 & $\circlearrowleft$ & $0 \leftarrow$ \\
\hline 0 & $\leftarrow$ & $0 \downarrow$ \\
\hline & & $\circlearrowleft$ \\
\hline
\end{tabular}

In this example, one has $d=|I|=|J|=4$, so that that $\operatorname{bit}(d)=3, N=\operatorname{bit}(N)=1$ and $n=4$. However, it is easy to see that the bounds obtained in Section 3 do not depend on $n$, but rather on $\bar{n}$, the number of non-absorbing states. We focus on the initial state $k=1$.

Computation of a 0.05-approximation of $v^{1}$. First of all, $2^{-5}<0.05<2^{-4}$, so we need our algorithm to compute a $2^{-r}$-approximation with $r=5$. By Proposition 3.3 , the defining polynomial of $v^{1}$ is of degree at most $\bar{n} d=8$, and the bit-size of its coefficients are bounded by $C:=4 \varphi(\bar{n}, d, N, 0)=192$. By Algorithm 2 approx bis, all we need to do is set $\lambda_{r+1}:=2^{-C-(r+1) \bar{n} d}=2^{-240}$, and then run Algorithm 1 approx with input $\lambda_{r+1}$ and precision $r+1=6$. The latter will compute the value of the following $|I| \times|J|$ matrix

$$
W_{\lambda}^{1}(z)=\lambda^{2}\left(\begin{array}{cccc}
\lambda^{2}(1-z) & \lambda(1-z) & -\lambda(1-\lambda)-\lambda z & -\left(2 \lambda-\lambda^{2}\right) z \\
\lambda(1-z) & \lambda(1-z) & -\left(2 \lambda-\lambda^{2}\right) z & -(1-\lambda)^{2}-z \\
-\lambda(1-\lambda)-\lambda z & -\left(2 \lambda-\lambda^{2}\right) z & \lambda(1-z)-\lambda z & 1-\lambda-z \\
-\left(2 \lambda-\lambda^{2}\right) z & -(1-\lambda)^{2}-z & 1-\lambda-z & 1-\lambda-z
\end{array}\right)
$$

for $\lambda=2^{-240}$ and well-chosen $z$ in the set $\left\{m 2^{-6}, 0 \leq m \leq 2^{6}\right\}$. The output $u$ will then satisfy $\left|u-v_{\lambda}^{1}\right| \leq 2^{-6}$, and Theorem 3 gives then $\left|v_{\lambda}^{1}-v^{1}\right| \leq 2^{-6}$ so that $\left|u-v^{1}\right| \leq 2^{-5}$.

Computation of an exact expression for $v^{1}$. By Theorem 2, this can be done with Algorithm 2 exact, which starts by setting $C:=4 \varphi(\bar{n}, d, N, 0)=192, s:=$ $\left\lceil d^{2} / 2+(3 d+4) \log _{2}(d+1)+2 d C\right\rceil=1582$ and $r:=s\left\lceil\log _{2} 12 d\right\rceil=8836$, before calling Algorithm 2 approx bis with precision level $r+1$. The latter sets then $\lambda_{r+1}=$ $2^{-C-(r+1) \bar{n} d}=2^{-70880}$ and runs Algorithm 1 approx with input $\lambda=2^{-70880}$ and precision $r+1$. The output is denoted $u$, and satisfies $\left|u-v^{1}\right| \leq 2^{-8836}$. Finally, one needs to run the $K K L$ algorithm with inputs $d, C$ and $u$ to obtain the defining polynomial $Q$ of $v^{1}$, which together with $u$ and $u+2^{-8836}$ give the desired exact expression for $v^{1}$.

\section{Acknowledgements}

I am very much indebted to Krishnendu Chatterjee for his useful comments and time, and to Kristoffer Hansen for his insight and advice. I am also thankful to the comments of the anonymous referees of the journal, which have greatly contributed in the presentation and organization of the results. Finally, I gratefully acknowledge the support of the French National Research Agency, under grant ANR CIGNE (ANR-15-CE380007-01), and the support of the Cowles Foundation at Yale University. 


\section{References}

[1] L. Attia and M. Oliu-Barton, A formula for the value of a stochastic game, ArXiv: 1809.06102. Proceedings of the National Academy of Sciences of the United States of America, 2020.

[2] S. Basu, R. Pollack, and M.-F. Roy, Algorithms in real algebraic geometry, vol. 10, Springer Science \& Business Media, 2007.

[3] T. Bewley and E. Kohlberg, The asymptotic theory of stochastic games, Mathematics of Operations Research 1 (1976), 197-208.

[4] _ On stochastic games with stationary optimal strategies, Mathematics of Operations Research 3 (1978), 104-125.

[5] D. Blackwell and T.S. Ferguson, The Big Match, Annals of Mathematical Statistics 39 (1968), 159-163.

[6] A. Cauchy, Calcul des indices des fonctions, Journal de l'École Polytechnique 15 (1832), no. 25, 176-229.

[7] K. Chatterjee, R. Majumdar, and T.A. Henzinger, Stochastic limit-average games are in EXPTIME, International Journal of Game Theory 37 (2008), 219-234.

[8] K. Etessami and M. Yannakakis, Recursive concurrent stochastic games, International Colloquium on Automata, Languages and Programming. Part II, Lecture Notes in Computer Science, vol. 4052, Springer, Berlin, 2006, pp. 324-335.

[9] K.A. Hansen, R. Ibsen-Jensen, and P.B. Miltersen, The complexity of solving reachability games using value and strategy iteration, International Computer Science Symposium in Russia, Springer, 2011, pp. 77-90.

[10] K.A. Hansen, M. Koucký, N. Lauritzen, P.B. Miltersen, and E.P. Tsigaridas, Exact algorithms for solving stochastic games, Proc. 43rd Annual ACM Symposium on Theory of Computing, 2011, pp. 205-214.

[11] T.A. Henzinger, L. de Alfaro, and K. Chatterjee, Strategy improvement for concurrent reachability games, Third International Conference on the Quantitative Evaluation of Systems (QEST), IEEE, 2006, pp. 291-300.

[12] R. Kannan, A. K. Lenstra, and L. Lovász, Polynomial factorization and nonrandomness of bits of algebraic and some transcendental numbers, Mathematics of Computation 50 (1988), 235-250.

[13] N. Karmarkar, A new polynomial-time algorithm for linear programming, Proc. 16th Annual ACM Symposium on Theory of Computing, ACM, 1984, pp. 302311.

[14] E. Kohlberg, Repeated games with absorbing states, Annals of Statistics 2 (1974), 724-738.

[15] K. Mahler, An inequality for the discriminant of a polynomial, The Michigan Mathematical Journal 11 (1964), no. 3, 257-262.

[16] J.-F. Mertens and A. Neyman, Stochastic games, International Journal of Game Theory 10 (1981), 53-66. 
[17] M. Mignotte, An inequality about factors of polynomials, Mathematics of Computation 28 (1974), 1153-1157.

[18] S. Rao, R. Chandrasekaran, and K.P.K. Nair, Algorithms for discounted stochastic games, Journal of Optimization Theory and Applications 11 (1973), 627-637.

[19] J. Renault, A tutorial on zero-sum stochastic games, ArXiv:1905.06577, 2019.

[20] L.S. Shapley, Stochastic games, Proceedings of the National Academy of Sciences of the United States of America 39 (1953), 1095-1100.

[21] L.S. Shapley and R.N. Snow, Basic solutions of discrete games, Contributions to the Theory of Games, Vol. I (H.W. Kuhn and A.W. Tucker, eds.), Annals of Mathematics Studies, vol. 24, Princeton University Press, Princeton, NJ, 1950, pp. 27-35.

[22] E. Solan and N. Vieille, Computing uniformly optimal strategies in two-player stochastic games, Economic Theory 42 (2010), 237-253.

[23] S. Sorin, A First Course on Zero-Sum Repeated Games, vol. 37, Springer Science \& Business Media, 2002. 